\title{
Posterior sternoclavicular dislocation in a rugby player as a cause of silent vascular compromise: a case report
}

\section{A H Mirza, K Alam, A Ali}

Br J Sports Med 2005;39:e28 (http://www.bjsportmed.com/cgi/content/full/39/5/e28). doi: 10.1136/bjsm.2004.014852

\begin{abstract}
Background: Approximately 120 cases of posterior sternoclavicular joint (SCJ) dislocation have been documented in the medical literature since it was first described in 1824 by Sir Astley Cooper, a statistic which underlies its relative rarity. It is associated with high energy trauma, and although it may present innocently enough, it is a potentially life threatening injury.

Case and Results: We describe a case in which there was no clinical evidence of complication, although CT imaging revealed complete obstruction of the brachiocephalic vein and impingement of the aorta. This required open reduction and a novel fixation technique was employed. The reduction was stable at 8 month follow up appointment as evidenced by $\mathrm{CT}$ scan.

Conclusions: We acknowledge that this type of complication is well recognised but emphasise that it should not be managed complacently. A high index of suspicion is required to determine the presence of serious complications in this type of injury, which may manifest insidiously.
\end{abstract}

\footnotetext{
A 19 year old male was admitted with a history of being injured whilst playing rugby. During the match he was holding the ball in his left arm and was tackled from behind. The opposing player wrapped his arm around the patient's left shoulder from behind. They both then fell forwards, with the patient holding the ball in his left arm and the weight of the opposing player on top of him. The result was an audible cracking sound and immediate pain in his left sternoclavicular area.

When he was seen in the accident and emergency department of the hospital, he was unable to move his left shoulder. There was a visible deformity and a palpable deficiency at the left sternoclavicular joint (SCJ). There was also marked tenderness at the medial end of the clavicle. Importantly, pulses in both arms were normal and his heart rate was 76. Respiratory examination was also normal and there was no stridor or dyspnoea. There was no dysphagia. Sensation and movement of both hands and fingers were normal. However several hours later the patient complained of being unable to take deep breaths.

The suspected diagnosis was posterior SCJ dislocation and investigations were carried out urgently. Chest radiography was inconclusive, the only abnormality being asymmetry of the SCJs. Oblique views of the clavicle were unhelpful. The diagnosis was confirmed by a contrast enhanced CT scan, at which it was clearly seen that "...the medial clavicle is dislocated infero-medially. It abuts the aortic arch but no leakage of contrast is seen. After injection of contrast into the left hand, no contrast was seen in the mediastinal structures suggesting obstruction of the left brachiocephalic vein". A three-dimensional (3D) reconstruction view is reproduced in fig 1 .
}

In view of this diagnosis, the patient was taken to theatre with the intention of closed reduction in the first instance, although he gave his consent for an open procedure. At operation it was found to be impossible to reduce the joint by closed means. A small incision over the medial end of the clavicle was made and the posterior dislocation was clearly seen. Once reduced, the joint was stabilised with a bone suture utilising non-absorbable suture material (figs 2 and 3 ).

The patient was discharged home on the second postoperative day. He was given a poly-sling in order to immobilise his shoulder for a period of 3-4 weeks to allow the ligaments to heal. An outpatient follow up CT scan was arranged at 8 months following injury, at which time satisfactory maintenance of reduction was seen (fig 4).

\section{DISCUSSION}

The SCJ is formed where the medial end of the clavicle articulates with the manubrial part of the sternum. It is a relatively incongruous joint, gaining stability from nearby ligaments which are much stronger posteriorly. It is considered to be a gliding joint, although only a ball and socket type joint matches the range of motion possible at the SCJ. The joint is most mobile in younger patients, which explains the preponderance of SCJ dislocation in this age group.

Contributing to mobility at the joint is the intra-articular disc, which divides the joint into two compartments. Angulation at the joint can reach $60^{\circ}$ in extremes of shoulder girdle movement. Immediately behind the joint on the right side lies the brachiocephalic vein. On the left side are the common carotid artery and subclavian vein with the subclavian artery lying posterior to these vessels.

\section{Mechanism of injury}

Although a few cases of atraumatic posterior dislocation and subluxation have been reported, a significant history of compressive or violent force is usually required to cause such an injury. ${ }^{1}$ Such a force is usually needed either directly over the medial clavicle or to the posterolateral aspect of the shoulder. Contact sports (especially the martial arts, American Football, and rugby) and motorcycle injuries are the commonest causes worldwide. ${ }^{23}$

\section{Diagnosis}

The invariable symptom of the injury is pain in the affected shoulder. The patient will be unable to move the affected shoulder and may complain of pain on moving the contralateral shoulder. A depression may be seen and palpated on the affected side.

Radiological confirmation can sometimes be difficult. Standard AP views may simply show asymmetry, and various oblique views may be unhelpful. Other views suggested

Abbreviations: SCJ, sternoclavicular joint 


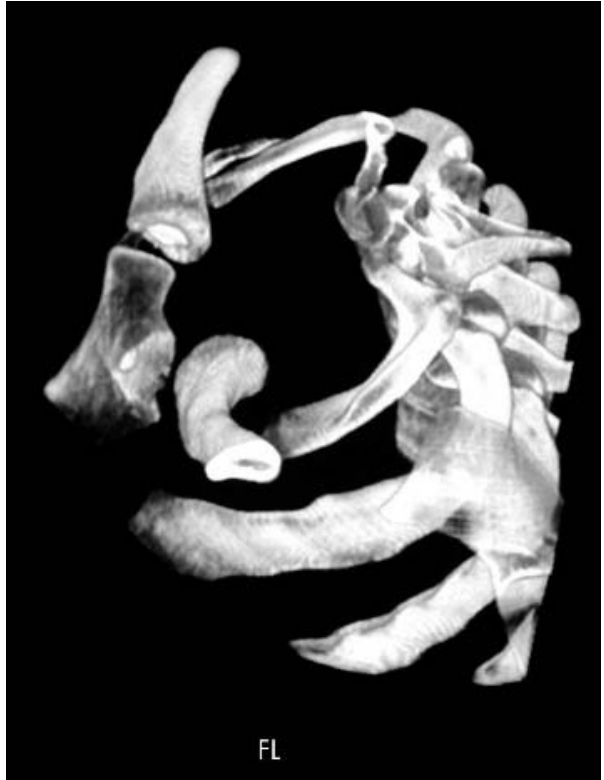

Figure $13 D$ reconstruction view clearly showing dislocation.

include the "serendipity" (cephalo-caudal) view. Successful use of ultrasound scanning has been reported although this requires operator experience. ${ }^{4}$

In our opinion CT scanning in stable patients is the ideal method since it can also delineate related injuries, and this has been well described in the literature. ${ }^{125}$ In the event of suspected vascular injury, intravenous contrast may be used to enhance CT interpretation. Angiography has also been employed both pre- and post-reduction, though due to its invasive nature is only useful for selected cases.

\section{Complications}

Rarely, the injury can present as potentially life threatening respiratory obstruction with dyspnoea in a young patient. This should be dealt with by immediate reduction using towel clips under local anaesthesia in the accident and emergency department itself. Other life threatening complications, which may occur in up to $25 \%$ of cases, include haemopneumothorax, tracheal damage, great vessel injury or laceration, and damage to the larynx with vocal cord palsy. ${ }^{2}$ Occasionally the patient may also complain of dysphagia or a choking sensation. Late complications include osteomyelitis of the clavicle and oesophageal fistula. ${ }^{3}$

\section{Treatment}

Some authorities consider these types of injury to in fact be epiphyseal fractures rather than true dislocations, especially in patients under 25 years of age. The treatment should not

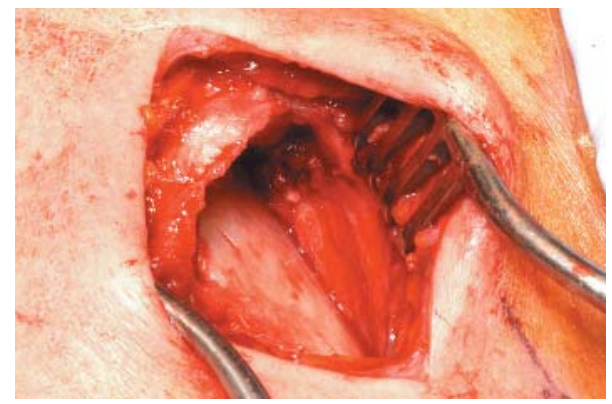

Figure 2 Operation photograph showing the dislocated SCJ.

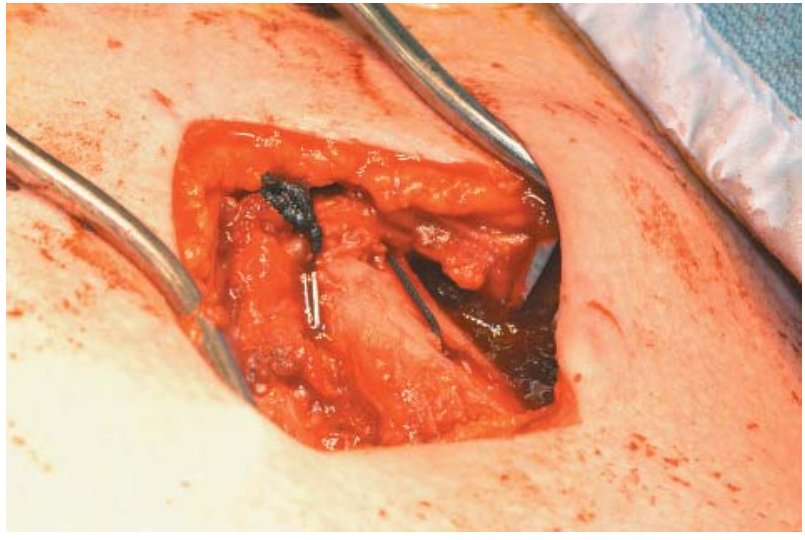

Figure 3 Operation photograph showing the reduced SCJ with bone anchor.

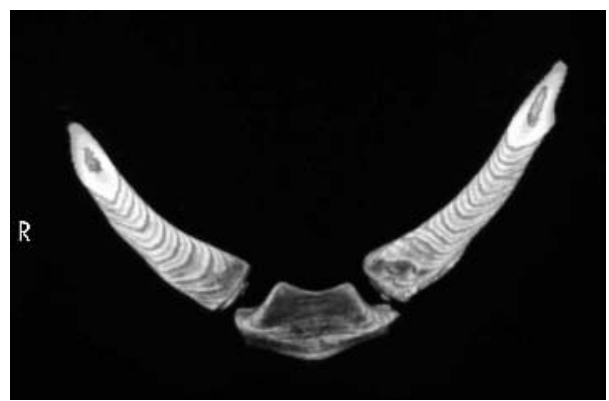

Figure $43 \mathrm{D}$ reconstruction view at 8 month follow up appointment. The joint reduction is stable.

differ and reduction is advised in all cases. This should be by closed means if at all possible, and the stability of the reduction should be assessed. If closed reduction is impossible or if the joint is unstable, then an open reduction is advised.

Internal fixation is not recommended, although the technique of a bone suture documented above is a novel technique, affording stability in the short term. In extreme cases, where open reduction has not been possible, resection of the medial clavicle has been documented, although this is undesirable. $^{6}$

\section{What is already known on this topic}

Posterior sternoclavicular joint dislocation is a rare injury, with potentially fatal sequelae. Delays in diagnosis or treatment can have serious implications.

\section{What this study adds}

Some serious complications of posterior sternoclavicular joint dislocation may not be immediately apparent, and a degree of vigilance and suspicion are required. If necessary, open reduction may be augmented by the use of a non-absorbable bone anchor type of suture. 


\section{Authors' affiliations}

A H Mirza, K Alam, A Ali, University Hospitals Birmingham, Birmingham, UK

Competing interests: none declared.

The patient described in this report consents to these details being published.

Correspondence to: Aun H Mirza, University Hospitals Birmingham, Orthopaedics and Trauma, Birmingham, UK; aunmirza@hotmail.com

Revised version received 19 December 2004

Accepted for publication 11 January 2005

\section{REFERENCES}

1 Pearson MR, Leonard RB. Posterior sternoclavicular dislocation: a case report. J Emerg Med 1994;12(6):783-7.

2 Marker LB, Klareskov B. Posterior sternoclavicular dislocation: an American Football injury. Br J Sports Med 1996:30:71-2.

3 Hidalgo Ovejero AM, Garcia Mata S, Sanchez Villares JJ, et al. Posterior sternoclavicular dislocation. Report of two cases. Acta Orthop Belg 2003:69(2): 188-92.

4 Benson LS, Donaldson JS, Carroll NC. Use of ultrasound in management of posterior sternoclavicular dislocation. J Ultrasound Med 1991;10:115-8.

5 Leighton RK, Buhr AJ, Sinclair AM. Posterior sternoclavicular dislocations. Can J Surg 1986;29(2): 104-6.

6 Santos GH, Sidotti E. Sternoclavicular dislocation (letter). J Cardiothorac Cardiovasc Surg 1990;99(6):1114-5. 